\title{
The Analysis and Review of the Study Trends of Empathy Using Arts Media in Youth Counseling
}

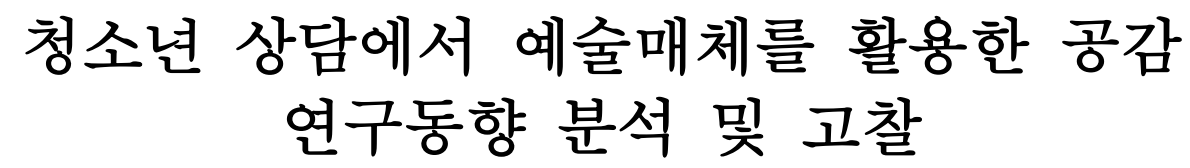

Sun Young Park ${ }^{1}$

박선영 ${ }^{1}$

${ }^{1}$ Ph. D, Integrative Arts Therapy, Dongduk Women's University, South Korea, cap1224@naver.com

\begin{abstract}
The purpose of this study is to analyze and consider research trends on empathy theory based on studies using various arts media in the field of youth counseling. First, the research trend of empathy theory using art media in youth counseling is the subject word of 'Adolescent Empathy' and 'Adolescent Empathy Arts among academic journal papers registered in KCI on RISS (Academic Research Information Service) from 2010 to April 2021 a total of 78 articles were retrieved. Among them, 'Adolescent Empathy' and 'Adolescent Empathy Arts were searched for in 9 academic journal areas, and 32 papers containing 'Empathy' in the thesis title were finally selected and analyzed. As a result of the study, the status of adolescent empathy papers by year was 3 in 2010, 3 in 2012, 3 in 2013, 1 in 2014, 6 in 2015, 3 in 2017, 7 in 2018, 4 in 2019, and 2020 was reported as one in a year and one in 2021, The age division of the group was confirmed as early adolescents, middle school students, high school students, middle and high school students, late adolescents, and adolescent-related adults. Also, as for group design, single-experimental group studies were the most common, and there were many studies using quantitative analysis. Among them, research related to arts therapy using arts media was found to be very incomplete with a total of 4 out of 32 papers. Therefore, based on the research result that the number of empathy studies using art media in youth counseling is very limited and insufficient, future research is expected to be utilized as basic data to verify the effectiveness of arts as a therapeutic tool.
\end{abstract}

Keywords: Youth Counseling, Arts Medium, Empathy, Therapeutic Function of Empathy

요약: 본 연구의 목적은 청소년 상담 분야에서 다양한 예술매체를 활용한 연구들을 기초로 하여 공감에 대한 연구동향을 분석 고찰하는 것이다. 먼저 청소년 상담에서 예술매체를 활용한 공감의 연구동향은 2010년부터 2021년 4월까지 RISS(학술연구정보 서비스)에 KCI 등재된 학술지 논문 중 ‘청소년 공감' , ‘청소년 공감 예술' 이라는 주제어 키워드로 검색된 논문은 총 78 편이었다. 이 중 9 개의 학술지 영역에서 ‘청소년 공감' , ‘청소년 공감 예술' 을 검색하여 논문 제목에서 '공감’ 이 포함된 논문 32편을 최종 선정하여 분석하였다. 연구 결과, 연도별 청소년 공감 논문 현황은 2010년 3편, 2012년 3편, 2013년 3편, 2014년 1편, 2015년 6편, 2017년 3편, 2018년 7편 2019년 4편, 2020년 1편 2021년 1편으로 보고 되었으며, 집단의 연령 구분을 초기 청소년, 중학생, 고등학생, 중-고등학생, 후기 청소년, 청소년 관련 성인으로 확인되었다. 또한 집단 설계는 단일 실험 집단 연구가 가장 많았으며, 양적 분석을 활용한 연구가 많이 나타났다. 그중 예술매체를 활용한 예술치료 관련 연구는

Received: June 10, 2021; $1^{\text {st }}$ Review Result: July 26, 2021; $2^{\text {nd }}$ Review Result: September 14, 2021

Accepted: October 31, 2021 
전체 논문 32 편 중 총 4편으로 매우 미비하였음을 확인할 수 있었다. 따라서 청소년 상담에서 예술매체를 활용한 공감 연구 수는 매우 한정적이고, 충분하지 않다는 연구결과를 기초로 하여 추후 연구에서는 치료적 도구로서 예술이 지닌 효과성을 검증하기 위한 기초자료로써 활용되기를 기대한다.

핵심어: 청소년상담, 예술매체, 공감, 공감의 치료적 기능

\section{1. 서론}

청소년의 친사회적 행동을 이해하기 위해 상담학과 심리학, 예술 치료학 등에서 널리 활용되는 개념 중 하나가 공감이다. 공감의 사전적 의미는 '타인의 감정과 의견, 주장에 대하여 자기 자신도 그렇다고 느끼는 정서와 기분' 을 뜻한다[1]. 즉 청소년이 학교생활, 또래 관계, 학업, 문화, 비행 등의 큰 영향을 미치는 중요한 공감 능력이다. 청소년 상담에서 다양한 예술매체를 치료적 도구로 활용하는 공감 연구가 증가하고 있는 추세이며, 공감을 정서적, 인지적 측면과 표현적 측면인 '의사소통' 과 '사회적 기술' 의 요소도 고려하는 치료적 공감의 개입을 깊게 살펴볼 필요가 있다[1]. 청소년 상담에서 '공감' 이라는 용어는 19세기 말에 처음 언급되었으며, 'Empathy' , 'sympathy' 라는 용어로 혼용되어 사용되어 오고 있다. 그리스어 'empatheir' 의 의미가 담겨있으며, 'Einfühlung' 라는 독일어 용어로 처음 사용하였다. 이는 '들어가서 느낀다' 라는 함축의 의미가 내포되어 있다[2].

다양한 학문분야에서 예술매체를 활용하는 것은 공감의 비언어적인 의사소통 수단이라고 할 수 있으며, 예술의 창조성, 은유와 상징 그리고 상상의 경험들은 예술매체가 지닌 치유의 힘으로 볼 수 있다. 즉 공감은 청소년 상담 과정에서 매우 중요한 요인으로 작용되고, 타인의 감정과 정서를 인식하며, 이에 대한 적절한 공감은 내담자의 보다 깊은 무의식 세계를 들여다볼 수 있는 치료적 도구로 여겨진다.

공감은 청소년의 심리적 안정감에도 지대한 영향을 미치는데, 이는 청소년의 또래관계 및 대인관계, 학업성취, 학교생활, 비행 문제 등과 같은 변인과 깊은 연관성이 있으며, 공감이 높을수록 청소년의 학업성취와 교우관계 및 자기조절 능력 등에 긍정적인 영향을 미치는 것으로 나타났다[3][4]. 반면 예술매체를 활용한 공감에 관한 연구는 미비하게 이루어지고 있으며, 청소년 상담에서 공감에 대한 연구가 필요한 실정이다.

따라서 본 연구는 청소년 상담 분야에서 다양한 예술매체(미술, 연극, 음악, 영화, 사진 등)를 활용한 공감에 대한 견해들을 살펴본 이후 연구동향을 분석하여 고찰하고자 한다. 이러한 연구결과는 청소년 상담분야의 전문가들이 임상현장에서 예술매체를 적용한 공감 치료를 시행하는 데 있어서 치료적 방향성에 도움이 되는 기초자료로서 활용되기를 기대한다.

\section{2. 이론적 배경}

\section{1 청소년 상담에서 공감 이해}

청소년 상담에서 공감은 청소년들이 경험하는 일상생활에서의 다양한 사회적 대인관계 및 또래관계 속에서 긍정적인 역할을 하고 있다고 해도 과언이 아니다. 대인관계 안에서의 
상호작용 촉진은 또래 문화의 다양성을 공감적 지지와 정서적인 지지로 타인의 마음을 이해하는 반응으로 작용되는 능력을 말한다[5]. 원만한 관계를 맺기 위한 능력 중 하나인 공감은 청소년의 친사회적 행동을 촉진시키고, 갈등 해소와 대인관계 증진 능력을 향상시키는 필수적인 핵심 요소로 작용된다[6]. 공감은 청소년 또래관계에서 소외된 감정과 관계에서 일어나는 단절의 경험은 많은 문제를 극복하는 보호요인으로 긍정적인 영향을 미치고 있다[7]. 또한 공감이 청소년의 친사회적인 행동과 이타적 행동, 사회성, 대인 관계 능력, 자기조절 능력 등의 연구결과에 높은 영향을 미치며, 최근에는 공감을 정서적, 인지적, 표현적 요소를 모두 포괄하는 개념으로 정의하고 있다[6][8].

여러 학자들에 의해 공감이 미치는 다양한 견해의 연구들이 발전해 왔다. 국외의 공감 연구를 살펴보면, Rogers[9]는 공감을 '내담자의 감정을 반영하는 것' 이라고 강조하면서 공감적 태도를 보고하였다. Dovidio[10]는 공감을 타인의 감정을 있는 그대로 이해하는 인지적 태도의 능력으로 보았으며, 공감은 자신의 행동과 관련하여 타인들이 가지게 되는 기대를 자신에게 제시하는 이타적 행동의 선행되는 원인으로 강조하였다. 특히 Mackay[11]는 공감을 세 가지 관점으로 행동과 성격 및 정서로 보는 관점이 포함된다고 언급하였다. 이러한 연구들을 통해 공감은 청소년 상담에서 가장 기본적인 태도이자 상담 성과에 중요한 요인이라는 점을 확인할 수 있었다.

국내에서 공감의 연구를 살펴보면, 정명선[12]은 공감 요인을 다섯 가지로 '정서적 개입, 상상적 공감, 정서 감염, 낮선 타인의 감정 이해, 공감적 관심 으로 명명하였고, 홍예영과 김유숙[6]은 공감 요인을 세 가지로 구분하여 '표현적, 인지적, 정서적' 표현으로 규명하였다. 또한 김윤희와 김진숙의 연구[13]에서는 공감 요인을 인지적 요소로

'역할 수용 및 감정 파악'으로, 정서적 요소는 '감정 공명 및 대리 감정'으로, 그리고 태도적 요소는 '진정성 및 경청' 으로 제시하였다. 마지막으로 허순향의 연구[14]에 의하면 공감 요인을 인지적, 정서적, 행동적 공감의 세 가지 요인으로 분류하였고, 인지적 공감은 '사고 조절 및 관점 수용 능력' 으로 정서적 공감에서는 '감정 표현 및 감정 몰입 능력' 으로 행동적 공감에서는 ‘의사소통 및 관계 형성 능력' 으로 설명하였다. 즉 공감은 정서적, 인지적, 행동적 측면과 사회적 공감까지 포함된 청소년들에게 필요한 다차원적인 개념의 요인으로 이해할 수 있다. 특히, 청소년 상담에서 진정한 공감은 내담자의 사고와 감정을 있는 그대로 느끼며, 지금의 상황을 이해하는 체험의 장을 마련할 필요가 있다[8]. 이는 청소년 상담에서 예술매체를 활용한 공감 프로그램의 체험 활동을 통하여 실질적으로 그들이 경험하는 여러 가지 사회적 공감 능력을 축적해 나아 갈 수 있을 것이다.

공감은 크게 두 가지로 대인관계 및 사회적 공감으로 나눌 수 있다. 대인관계 공감에서는 정서적, 인지적, 행동적 세 가지 요인으로 구분되며, 사회적 공감에서는 지역사회, 문화, 개인까지 포함한 사회적 책임을 포함한다. 첫 번째 대인관계 공감의 정서적 요인은 타인의 욕구와 공감적 배려 그리고 공감 표현까지 대인관계에서 타인과의 감정적인 연결성으로 이어지며,[6] 공감의 인지적 요인은 타인의 표정이나 비언어적 단서를 이해하기 위해 대인관계의 민감성이 요구된다[8]. 타인의 상황적, 정신적, 감정적 현상을 이해하는 것은 정신적 유연성으로 작용되며[15], 공감의 행동적 요인은 타인의 입장에서 서서 관찰할 수 있는 표현의 행동이다. 즉 타인에게 도움을 주고자 하는 정서적 상황과 상태의 동기유발의 행동을 취하는 개인의 의식적 행동과 정서적 반응을 포함한다[16].

두 번째 사회적 공감에서는 맥락적 이해와 거시적 관점 수용의 두 가지 개념으로 구분하여 제시하였다. 맥락적 이해는 정치적, 경제적, 사회적 제도가 그 사회구성원의 
개인과 집단에게 주는 부정적 영향을 체계적으로 이해하는 능력을 의미한다. 지역사회 및 문화 등에 대한 공감을 조사하고, 사회적 현상 안에서 맥락적 이해의 공감을 검토하여 사회적 책임과 정의까지 포함된 의미로 제공된다[17]. 공감의 구성요인은 다음 [표 1]과 같다.

$$
\text { [표 1] 공감의 구성요인 }
$$

[Table 1] Components of Empathy

\begin{tabular}{|c|c|c|c|}
\hline Division & Comp & lent & Contents \\
\hline \multirow{5}{*}{$\begin{array}{c}\text { Interpersonal } \\
\text { empathy }\end{array}$} & Emotional factor & $\begin{array}{l}\text { Emotional } \\
\text { reaction }\end{array}$ & $\begin{array}{l}\text { * Neurobiological capacity to be cultivated and improved } \\
\text { The ability to reflect and accurately identify the feelings } \\
\text { of others } \\
\text { Individual's involuntary physiological reactions and } \\
\text { emotions when observing or listening to others, } \\
\text { watching movies or reading books } \\
\text { Reflects emotional sharing and emotional congruence }\end{array}$ \\
\hline & \multirow{3}{*}{ Cognitive factor } & Self-awareness & $\begin{array}{l}\text { * The ability to temporarily identify with someone without } \\
\text { confusion between themselves and others } \\
\text { The ability to maintain appropriate boundaries between } \\
\text { personal emotions, thoughts and other people's } \\
\text { emotions, and thoughts. }\end{array}$ \\
\hline & & $\begin{array}{l}\text { Perspective } \\
\text { acceptance }\end{array}$ & $\begin{array}{l}\text { * The ability to be mentally flexible (imagining the } \\
\text { situation of others from the inside, from the perspective } \\
\text { of others) } \\
\text { \& Open to different views }\end{array}$ \\
\hline & & $\begin{array}{c}\text { Emotional } \\
\text { control }\end{array}$ & $\begin{array}{l}\text { * The ability to change or control one's emotional } \\
\text { experiences for reflected emotions } \\
\text { * The observer is not overwhelmed by the infectious side } \\
\text { of emotional sharing, meaning avoiding personal } \\
\text { suffering. }\end{array}$ \\
\hline & Behavioral factors & $\begin{array}{l}\text { Empathetic } \\
\text { attitude }\end{array}$ & $\begin{array}{l}\# \quad \begin{array}{l}\text { A socially responsible, empathetic belief set } \\
\text { Those who equate beliefs are supportive of interventions } \\
\text { to address poverty and are more likely to take personal } \\
\text { action }\end{array} \\
\text { \# } \begin{array}{l}\text { Developed a Empathy attitude (EAS) as a proxy measure } \\
\text { of conscious decision-making }\end{array} \\
\text { To support or equate socially responsible empathetic } \\
\text { attitudes and beliefs with those who talk to them } \\
\text { People who are close to empathy have the premise that } \\
\text { they are more inclined to act or support empathy }\end{array}$ \\
\hline \multirow[t]{2}{*}{$\begin{array}{c}\text { Social } \\
\text { empathy }\end{array}$} & \multirow[t]{2}{*}{$\begin{array}{l}\text { Social justice } \\
\text { responsibility }\end{array}$} & $\begin{array}{c}\text { Contextual } \\
\text { understanding }\end{array}$ & $\begin{array}{l}\text { * The ability to understand the impact of historical } \\
\text { background exposure and psychological disorders on } \\
\text { others, built on social, political, and economic systems } \\
\text { The ability to assess the situation of social groups in each } \\
\text { culture and the understanding of the social context are } \\
\text { important in empathy } \\
\text { Improving social relationships through the reduction of } \\
\text { stereotypes and prejudices } \\
\text { A contextual understanding of systemic disability is } \\
\text { important to understand the life experience of yourself } \\
\text { and other groups }\end{array}$ \\
\hline & & $\begin{array}{l}\text { Accepting a } \\
\text { macro } \\
\text { perspective }\end{array}$ & $\begin{array}{l}\text { * Living as part of another social group is the ability to } \\
\text { process cognitively } \\
\text { * Ability to get into other people's lives }\end{array}$ \\
\hline
\end{tabular}




\section{2 청소년 상담에서 예술매체를 활용한 치료적 기능으로서의 공감}

청소년 상담에서 예술매체는 치료적 기능으로서의 도구이다. 예술매체는 미술, 연극, 음악, 영화, 사진, 무용, 동작, 문학 등의 예술치료 체험 안에서 오감을 자극하고 다양한 정서와 감각을 통하여 내면의 무의식을 드러낸다. 비언어적 수단인 상징적 표현으로 자기이해를 촉진시킨다[18]. 예술치료가 예술행위로 자기표현을 증진시키고, 억압된 감정을 발산시키는데 유용하며, 자기표현의 수단인 행위로써 표현 자체에서 자기 치유의 속성을 지닌다[19]. 예술치료에서의 창의성과 즉흥성, 놀이성 등이 발현되는 심리적 기능에 관한 경험적 근거를 마련할 수 있으며, 예술치료 작업으로 억압된 감정 발산을 통하여 정서가 치유되고 무의식을 시각화하여 감정을 표현할 수 있는 치료적인 효과성은 예술이 지닌 힘이다. 또한 창조적인 예술제작 과정을 바탕으로 상징적인 언어표현이 가능하다[20].

통합예술치료적 관점에서 다양한 예술매체별 치료적 기제의 기능을 살펴보면, 연극 매체는 안전한 공간에서 즉흥성, 자발성, 창조성을 발현하여 심리적 거리 조절의 분리와 밀착의 균형을 이룬 상태를 의미한다. 다양한 역할연기를 통하여 억압된 감정을 여러 방식으로 표현해보고, 상대방의 입장에 서서 느껴보며 생각해 보는 재현의 경험을 가질 수 있는 매개체이다. 미술 매체는 내면을 촉진시키고 심상의 상징화 작업이며, 창조적 예술 활동은 개인의 무의식 기억과 경험을 시각적 이미지로 형상화하는 치료적 매개체의 기능을 한다. 음악 매체는 악기와 소품 등의 소리가 자신을 표현하는 수단으로 기능하며, 리듬, 박자, 멜로디, 화음 등의 요소와 연합하여 음악적 심상을 창조적 역동적으로 유도하는 매개체의 가능을 가진다. 또한 영화매체는 동적인 스크린을 통하여 시각적, 청각적 자극으로 종합예술을 경험한다. 영화는 영화 감상과 표현 영화치료 두 가지 방식으로 구분된다. 이는 투사적 도구로서 영화 감상은 강력한 정서를 활성화시키고 영화 속 인물과 자신을 동일시하며, 갈등 상황과 문제해결방법 그리고 대처 방식의 효과를 다양하게 적용 가능하다[19]. 사진 매체는 카메라 렌즈를 통해 얻어진 사람, 사물 등의 이미지를 일컫는다. 사진매체의 이미지는 다양한 정서들의 의미를 구축하고, 내적인 심상과 억압된 감정들의 욕구와 기억들이 사진이라는 매개체로 자극하는 역할 기능을 가지고 있다. 무용·동작 매체는 신체를 활용하여 언어로 표현하기 힘든 감정과 정서들을 움직임과 즉흥적 동작, 그리고 춤으로 육체와 정신을 통합시키는 매개체이다. 또한 자발적이고 자유로운 움직임과 감정 자극에 대한 완화로 작용되고, 자기표현과 내적 갈등을 승화시키며, 몰입의 과정은 감정적 변화를 촉진시키는 기능을 가진다. 문학 매체는 독서치료라고도 혼용하여 사용된다. 문학에서의 치료적 속성은 심리적 상황에서 자기이해 및 통찰을 기반으로 한 개개인의 창의적 체험과 기억을 통해 의식화 작업, 즉흥 글쓰기, 이야기 만들기, 낭독 등과 같은 내면화 체험에 치료적 효과성을 높일 수 있다[18].

이처럼 청소년 상담에서 공감 기회의 제공은 청소년을 이해하기 위한 필수적인 요인이며, 정서적인 지지와 또래로부터 관심을 받고 인정을 받을 수 있는 공감적 환경, 즉 사회화 과정의 공감 제공은 또래 문화 속에서 발현되는 관계 맺기에 효과적일 것이다[6]. 이때 예술매체는 청소년들이 격는 또래관계의 어려움, 학교생활 부적응, 부모와의 갈등, 성적 부진, 이성 고민 등 개인의 갈등을 조절하고 자기성장을 촉발시킬 수 있는 치료적 가능성의 필수적 대체도구로 실질적인 개입이 요구된다. 특히 청소년 상담에서 그들은 언어로 자신의 감정과 정서를 표현하는 데 어려움을 나타내고, 예술매체는 비언어적인 수단의 도구로 활용되는데, 이는 다양한 예술 활동을 통하여 
청소년들이 보이는 방어적 태도와 방어기제를 완화시킨다[20]. 즉 내면의 무의식적으로 억압된 갈등이나 고통을 자유롭게 창조적으로 표출하도록 돕는다. 이처럼 청소년 상담에서 예술매체를 활용한 공감은 청소년의 정서적 갈등을 조정하고 불안을 감소시키며, 대인관계와 사회성 기술의 발달 촉진과 공감 능력 향상 등은 임상현장에서 치료적 수단으로 적용되고 있다.

\section{3. 연구 방법}

\section{1 분석 논문 선정 기준}

본 연구는 청소년 상담에서 예술매체를 활용한 공감 연구동향을 살펴보고 고찰하였다. 2010년부터 2021년 4월까지 RISS(학술연구정보서비스)에 KCI 등재된 학술지 논문 중 '청소년 공감' , ‘청소년 공감 예술' 이라는 주제어 키워드로 검색된 논문은 총 78편이었다. 이 중 청소년학연구, 디지털융복합연구, 미래청소년학회지, 상담학연구, 청소년상담연구, 교정복지연구, 청소년복지연구, 한국청소년연구, 교육혁신연구에 수록된 총 9개의 학술지 영역에서 '청소년 공감' , '청소년 공감 예술' 을 검색하여 논문 제목에서 ‘공감' 이 포함된 논문 32편이 최종적으로 분석 대상에 선정되었다.

\section{2 분석 기준}

본 연구는 청소년 상담에서 예술매체를 활용한 공감 연구동향을 살펴보고 고찰하고자 하였으며, 청소년 관련 공감 논문 발행 현황, 연구자, 연도, 연구 제목, 연구 주제어 구분, 집단 설계에 따른 연령 구분, 양적 연구와 질적 연구 구분, 연구 방법에 따른 공감 연구 구분, 예술매체를 활용 공감 연구 구분, 전체 논문 주제어에 따른 공감 키워드 구분, 공감 단어가 들어간 주제어 키워드를 구분하여 분석하였다. 또한 하소영[4]의 연구와 김희진과 이미선[21]의 선행연구 분류를 바탕으로 하위 범주를 다음과 같이 살펴보았다.

\subsection{1 발행 연도에 대한 분류}

최종 분석 논문에서 2010년부터 2021년 4월까지 발행되어 표기된 연도를 기준으로 분류를 실시하였다.

\subsection{2 연령에 대한 분류}

국가통계포털에 명시된 연령별 분류를 근거로 하였으며, 최종 분석 논문에 명시된 연령을 기준으로 초기 청소년, 중학생, 고등학생, 중-고등학생, 후기 청소년, 청소년 관련 성인(상담자 및 부모)으로 분류하였다.

\subsection{3 집단 설계에 대한 분류}

집단 설계에 대한 분류는 실험-통제 집단과 실험-비교 집단, 그리고 실험-비교-통제 집단 기준으로 분류하였고, 단일 집단(통제집단 또는 대조 집단 없이 구성된 실험집단 연구)으로 설계된 연구로 구분하였다. 


\subsection{4 연구자, 연도, 연구 제목, 연구 주제어에 대한 분류}

최종 분석된 논문에서 명시한 연구자, 연도, 연구 제목, 연구 주제어를 기준으로 분류하였다.

\subsection{5 청소년 공감에 대한 연구 방법 분류}

최종 분석된 논문에서 명시한 청소년 공감 연구 방법을 양적 연구와 질적 연구, 문헌 연구 기준으로 분류하였다.

\subsection{6 연구 방법에 따른 청소년 공감 연구 분류}

연구 방법을 기준으로 매개효과 연구, 문헌 연구, 현상학적 연구, 사례연구, 프로그램 개발연구, 프로그램 효과 연구, 척도지 개발연구로 구분하였다

\subsection{7 예술매체를 활용한 공감 연구에 대한 분류}

최종 분석된 논문에서 예술매체 주제어와 공감이 들어간 주제어로 구분하였다.

\section{2 .8 전체 논문 주제어에 대한 공감 키워드 분류}

최종 분석된 논문에서 전체 논문 주제어와 공감 키워드가 들어간 주제어로 구분하였다.

\subsection{9 공감 단어가 들어간 주제어에 대한 분류}

최종 분석된 논문에서 공감 단어가 들어간 주제어로 구분하였다.

\section{3 자료 처리}

본 연구의 자료 분석은 하소영[4]의 연구와 김희진과 이미선[21]의 연구를 바탕으로 안면타당도(Face validity) 검증을 사용하였으며, 범주에 따른 내용 분석을 위해 코딩 시트를 구성하였다. 코딩 파일 범주에 따라 자료 분석을 실시하였고, 자료 처리는 통계 프로그램 SPSS 21.0(IBM Corp., Armonk, NY, USA)을 이용하여 표본 수에 따른 백분율을 산출하였으며, 발행 현황, 연구자, 연도, 연구 제목, 연구 주제어, 집단 설계에 따른 연령, 연구 방법 등을 측정 변인 별로 교차 분석(Cross-tabulation Analysis)과 빈도 분석(Frequency Analysis)을 시행하였다.

\section{4. 연구결과}

\section{1 청소년 상담에서 예술매체를 활용한 공감 연구 동향}

청소년 상담 분야에서 예술매체를 활용한 공감 연구동향을 청소년 관련 공감 논문 발행 현황, 연구자, 연도, 연구 제목, 연구 주제어 구분, 집단 설계에 따른 연령 구분, 양적 연구와 질적 연구 구분, 연구 방법에 따른 공감 연구 구분, 예술매체를 활용 공감 연구 구분, 전체 논문 주제어에 따른 공감 키워드 구분, 공감 단어가 들어간 주제어에 따라 분석하였다. 연도별로 청소년 공감 논문 발행 현황은 다음 [표 2]와 같다. 
[표 2] 연도별로 청소년 공감 논문 발행 현황

[Table 2] Status of Youth Empathy Research Publication by Year

\begin{tabular}{c|c|c|c|c|c|c|c|c|c|c|c}
\hline Year & 2010 & 2012 & 2013 & 2014 & 2015 & 2017 & 2018 & 2019 & 2020 & 2021 & Total \\
\hline $\mathrm{N}$ & 3 & 3 & 3 & 1 & 6 & 3 & 7 & 4 & 1 & 1 & 32 \\
\hline$(\%)$ & 9.4 & 9.4 & 9.4 & 3.1 & 18.7 & 9.4 & 21.9 & 12.5 & 3.1 & 3.1 & 100.0 \\
\hline
\end{tabular}

'청소년 공감' , ‘청소년 공감 예술' 주제어 키워드로 2010년부터 2021년까지 연도별로 청소년 공감 논문 발행 현황을 살펴보면, 2010년 3편(9.4\%), 2012년 3편(9.4\%), 2013년 3편(9.4\%), 2014년 1편(3.1\%), 2015년 6편(18.7\%), 2017년 3편(9.4\%), 2018년에는 7편(21.9\%), 2019년 4편(12.5\%), 2020년 1편(3.1\%), 2021년 1편(3.1\%)으로 나타났다. 이에 2015년과 2018년에 청소년 공감과 관련된 변인의 연구 편수가 다른 연도에 비해 활발히 증가하였음을 확인하였다. 이러한 연구들 대부분은 공감이 기능하는 역할에 초점을 두어 다른 변인들과의 관계를 탐색하고 있으며, 공감이 기능하는 역할에 초점을 맞춘 정서적, 인지적, 행동적 요인과 사회적 공감까지 포함된 연구들이 이루어지고 있다. $\mathrm{KCI}$ 등재된 학술지를 중심으로 연구자, 연도, 연구 제목, 연구 주제어에 따른 분류는 다음 [표 3]과 같다.

[표 3] 연구자, 연도, 연구 제목, 연구 주제어에 따른 분류

[Table 3] Researchers, by Year, Research Title, Research-specific Control Classification

\begin{tabular}{|c|c|c|c|c|}
\hline No & Researcher & Year & Title & Key word \\
\hline 1 & $\begin{array}{l}\text { Yu-Sung } \\
\text { Kim, Hee- } \\
\text { Jung Min }\end{array}$ & 2021 & $\begin{array}{l}\text { "The Mediating Effects of Self-Efficacy } \\
\text { and Cognitive Empathy in the } \\
\text { Relationships between College Students' } \\
\text { Self-Compassion and Meta-Cognition" }\end{array}$ & $\begin{array}{l}\text { College Students, Cognitive } \\
\text { Empathy, Self-Compassion, Self- } \\
\text { Efficacy, Meta-Cognition, }\end{array}$ \\
\hline 2 & $\begin{array}{l}\text { Young-In } \\
\text { Kim }\end{array}$ & 2020 & $\begin{array}{c}\text { "Analysis of the structural relationship, } \\
\text { direct and mediating effects between the } \\
\text { multicultural experience, multicultural } \\
\text { attitudes, empathic ability and } \\
\text { multicultural behavior intention of youth } \\
\text { leaders" }\end{array}$ & $\begin{array}{l}\text { Youth leader, Empathic Ability, } \\
\text { Multicultural Experience, } \\
\text { Multicultural Attitude, Structural } \\
\text { Relationship, Multicultural } \\
\text { Behavior Intention }\end{array}$ \\
\hline 3 & Jin-Sook Jang & \multirow{3}{*}{2019} & $\begin{array}{l}\text { "Relationship between maternal empathy } \\
\text { perceived by adolescents and their } \\
\text { emotional intelligence : Mediating effect } \\
\text { of open mother and child communication" }\end{array}$ & $\begin{array}{l}\text { Emotionalintelligence, Maternal } \\
\text { Empathy Perceived by } \\
\text { Adolescents, Open } \\
\text { Communication Between Mother } \\
\text { and Child }\end{array}$ \\
\hline 4 & $\begin{array}{l}\text { Eom, Seo- } \\
\text { Young, Soo } \\
\text { Mi-Jang, } \\
\text { Seong-Hui } \\
\text { Heo }\end{array}$ & & $\begin{array}{c}\text { "The Development and Effects of the } \\
\text { Empathy Enhancement Program for } \\
\text { Females Adolescent with Sexual } \\
\text { Misconducts" }\end{array}$ & $\begin{array}{c}\text { Empathy enhancement, } \\
\text { Intervention Research, Female } \\
\text { Adolescent, Sexual Misconducts }\end{array}$ \\
\hline 5 & $\begin{array}{l}\text { Ju-Young } \\
\text { Woo, Min } \\
\text { Park }\end{array}$ & & $\begin{array}{l}\text { "Resilience and Empathy on the Relation } \\
\text { between Humanity and Friendship of the } \\
\text { Early Adolescents" }\end{array}$ & $\begin{array}{l}\text { Empathy, Early adolescents, } \\
\text { Humanity, Friendship, Resilience }\end{array}$ \\
\hline
\end{tabular}




\begin{tabular}{|c|c|c|c|c|}
\hline 6 & $\begin{array}{c}\text { Su-Ah Jo, } \\
\text { Ae-Sim Kim }\end{array}$ & & $\begin{array}{l}\text { "The Relationship between Receptive } \\
\text { Parenting Attitudes Perceived by } \\
\text { Adolescents and Anger Regulation in } \\
\text { Adolescents : The Mediating Effects of } \\
\text { Self-Soothing and Empathy" }\end{array}$ & $\begin{array}{l}\text { Empathy, Receptive Parenting } \\
\text { Attitudes, At-Risk Youthrece, } \\
\text { Anger Regulation, Self-Soothing }\end{array}$ \\
\hline 7 & $\begin{array}{l}\text { Seo-Yeon } \\
\text { Jung, Eun- } \\
\text { Young Park }\end{array}$ & \multirow{7}{*}{2018} & $\begin{array}{l}\text { "The Effects of Youth's Empathy on } \\
\text { Cyber-Bullying Behaviors : Focused on } \\
\text { The Mediating Effect of Youth's Self- } \\
\text { Control” }\end{array}$ & $\begin{array}{l}\text { Youth's Self-Control, Youth's } \\
\text { Empathy, Cyber-Bullying } \\
\text { Behaviors }\end{array}$ \\
\hline 8 & $\begin{array}{l}\text { Kyoung-Ok } \\
\text { Kang, Choon- } \\
\text { Bum Cho. } \\
\text { Jung-Hwa } \\
\text { Kim }\end{array}$ & & $\begin{array}{l}\text { "Effect of Parent-Child Communication on } \\
\text { the Cyber Violence Behavior:The Effects } \\
\text { of Adolescents on Empathy Parameters" }\end{array}$ & $\begin{array}{c}\text { Empathy Skills for Young People, } \\
\text { Communication Method Between } \\
\text { Parents and Children, Cyber } \\
\text { Bullying Behavior }\end{array}$ \\
\hline 9 & $\begin{array}{l}\text { Chang Seek } \\
\text { Lee, Ha } \\
\text { Young Jang }\end{array}$ & & $\begin{array}{l}\text { "Moderating Effect of Empathy on the } \\
\text { Relationship between Self-esteem and } \\
\text { Aggression of Adolescents" }\end{array}$ & $\begin{array}{l}\text { Empathy, Self-esteem, Aggression, } \\
\text { Adolescent, Moderating effect, } \\
\text { School violence }\end{array}$ \\
\hline 10 & $\begin{array}{l}\text { Ji-Min Kim, } \\
\text { Young-sik } \\
\text { Lim }\end{array}$ & & $\begin{array}{l}\text { "The influence on client's expectations } \\
\text { about counseling(EAC), perception of } \\
\text { adolescent about counselor's empathy, } \\
\text { negative emotion on working alliance in } \\
\text { early sessions in counseling" }\end{array}$ & $\begin{array}{l}\text { Expectation About Counseling, } \\
\text { Perception of Adolescent About } \\
\text { Counselor's Empathy, Working } \\
\text { Alliance, Negative emotion }\end{array}$ \\
\hline 11 & $\begin{array}{l}\text { Ji-Min Kim, } \\
\text { Young-sik } \\
\text { Lim }\end{array}$ & & $\begin{array}{l}\text { "The influence of negative emotional } \\
\text { contagion, job circumstances, and } \\
\text { compassion fatigue on burn-out amongst } \\
\text { public institution youth counselors" }\end{array}$ & $\begin{array}{l}\text { Negative Emotional Contagion, } \\
\text { The Public Institution, Youth } \\
\text { Counselor's, Compassion Fatigue, } \\
\text { Working Environment }\end{array}$ \\
\hline 12 & Sang-Jun Han & & $\begin{array}{l}\text { "A Study on Influence of Adolescents' } \\
\text { Participation Type/Degree in Recreational } \\
\text { Activities on Emotional Empathy" }\end{array}$ & $\begin{array}{l}\text { Emotional Empathy, Participation } \\
\text { Type, Adolescent, Recreational } \\
\text { Activities, Participation Degree }\end{array}$ \\
\hline 13 & $\begin{array}{l}\text { Min-Ju Shin, } \\
\text { Yong-Kook } \\
\text { Joo }\end{array}$ & & $\begin{array}{l}\text { "A Qualitative Case Study on Integrated } \\
\text { Art Therapy Program to Improve Empathy } \\
\text { Ability of Adolescents with } \\
\text { Developmental Disabilities" }\end{array}$ & $\begin{array}{l}\text { Empathy, Integrated Art Therapy } \\
\text { Program, Developmental } \\
\text { Disabilities Adolescent, } \\
\text { Qualitative Case Study }\end{array}$ \\
\hline 14 & $\begin{array}{l}\text { Sun-young } \\
\text { Park, Yong- } \\
\text { Joo Jo }\end{array}$ & \multirow{3}{*}{2017} & $\begin{array}{l}\text { "Item Analysis and Modification of a } \\
\text { Parental Empathy Inventory Perceived by } \\
\text { Adolescent Children Scale under Multiple } \\
\text { Item Response Theory" }\end{array}$ & $\begin{array}{l}\text { Excessive Emotional Reaction, } \\
\text { Parental Empathy Inventory } \\
\text { Perceived by Adolescent Children, } \\
\text { Generalized Partial Credit Model, } \\
\text { Polytomous item Response Theory }\end{array}$ \\
\hline 15 & $\begin{array}{l}\text { Sun-Hee } \\
\text { Park, Jeeh- } \\
\text { young Yu }\end{array}$ & & $\begin{array}{l}\text { "The Effects of Multicultural Experience } \\
\text { on Empathy in Adolescents: Focused on } \\
\text { Mediating Effect of Multicultural } \\
\text { Acceptance and Cultural Empathy" }\end{array}$ & $\begin{array}{l}\text { Empathy, Cultural Empathy, } \\
\text { Adolescents, Multicultural } \\
\text { Experience, Multicultural } \\
\text { Acceptance }\end{array}$ \\
\hline 16 & $\begin{array}{l}\text { Min-gyu } \\
\text { Kang, Yoon- } \\
\text { jung Choi }\end{array}$ & & $\begin{array}{l}\text { "The Effects of Brief Mindfulness } \\
\text { Meditation on Empathy and Self- } \\
\text { Regulation ability in the Perpetrators of } \\
\text { School Violence" }\end{array}$ & $\begin{array}{l}\text { Empathy Ability, Brief } \\
\text { Mindfulness Meditation, Empathy } \\
\text { Training Program, Self- } \\
\text { Regulation, Adolescents }\end{array}$ \\
\hline 17 & $\begin{array}{l}\text { Nam- Hee } \\
\text { Kweon, } \\
\text { Sang-In Nam }\end{array}$ & \multirow{3}{*}{2015} & $\begin{array}{l}\text { "Effect of parents` rearing attitude, } \\
\text { prosocial behavior and empathetic attitude } \\
\text { on adolescents` prosocial behavior: } \\
\text { Focused on mediating effect of } \\
\text { adolescents` emotional empathy" }\end{array}$ & $\begin{array}{c}\text { Adolescents` Prosocial Behavior, } \\
\text { Adolescents` Emotional Empathy, } \\
\text { Parents` Empathetic Attitude, } \\
\text { Parents` Prosocial Behavior, } \\
\text { Rearing Attitude }\end{array}$ \\
\hline 18 & $\begin{array}{l}\text { Ye-Young } \\
\text { Hong, Yoo- } \\
\text { Sook Kim }\end{array}$ & & $\begin{array}{l}\text { "Development and Validation of the } \\
\text { Adolescent Empathy Scale" }\end{array}$ & $\begin{array}{l}\text { Empathy Scale, Empathy of } \\
\text { Adolescents, Factor Analysis, } \\
\text { Validitr, Eliability }\end{array}$ \\
\hline 19 & $\begin{array}{l}\text { Mi-Kyong } \\
\text { Choi, Seo } \\
\text { Yeong Lee }\end{array}$ & & $\begin{array}{l}\text { "A Study on Empathy Ability, Awareness } \\
\text { \& Attitude Change through Empathy } \\
\text { Centered-School Violence Prevention } \\
\text { Education for Non-Adapted Student" }\end{array}$ & $\begin{array}{l}\text { School Maladjusted Adolescents, } \\
\text { School Violence Prevention } \\
\text { Program, Empathy Ability, School } \\
\text { Violence Awareness, Attitude of } \\
\text { School Violence }\end{array}$ \\
\hline
\end{tabular}




\begin{tabular}{|c|c|c|c|c|}
\hline 20 & $\begin{array}{l}\text { Ye-Hyun } \\
\text { Oh, , Jae- } \\
\text { Hong Jang }\end{array}$ & & $\begin{array}{l}\text { "A Phenomenological Research on } \\
\text { Perception of" }\end{array}$ & $\begin{array}{l}\text { Perceived Empathy, Adolescent, } \\
\text { Client, Phenomenological Method }\end{array}$ \\
\hline 21 & $\begin{array}{l}\text { In-Hyung } \\
\text { Kim }\end{array}$ & & $\begin{array}{l}\text { "The Influences of the Youth' Empathy } \\
\text { and Resilience on Violent Attitudes and } \\
\text { Problematic Behaviors in School Sports } \\
\text { Clubs Participation" }\end{array}$ & $\begin{array}{c}\text { School Sports Club, Problematic } \\
\text { Behaviors, Delinquency, } \\
\text { Aggressiveness }\end{array}$ \\
\hline 22 & $\begin{array}{l}\text { Seung-Ju Oh, } \\
\text { Se Min -Choi }\end{array}$ & & $\begin{array}{c}\text { "The Effects of Group Art Therapy Based } \\
\text { on Cognitive Behavior Theory on } \\
\text { Automatic Thought, and Empathy of } \\
\text { Juvenile Delinquents" }\end{array}$ & $\begin{array}{c}\text { Empathy, Group Art Therapy, } \\
\text { Juvenile Delinquents, Cognitive } \\
\text { Behavior Therapy, Automatic } \\
\text { Thought }\end{array}$ \\
\hline 23 & Jung-Mi Kim & 2014 & $\begin{array}{c}\text { "The Moderating Effect of Optimism and } \\
\text { Perceived Parental Empathy in the } \\
\text { Relation between School-related Stress } \\
\text { and Depression" }\end{array}$ & $\begin{array}{l}\text { Parental Empathy, Depression, } \\
\text { Optimism, Stress }\end{array}$ \\
\hline 24 & $\begin{array}{l}\text { Myoung-Suk } \\
\text { Hong, Joo- } \\
\text { Kyeong Eo }\end{array}$ & \multirow{3}{*}{2013} & $\begin{array}{l}\text { "The Effects of Narcissism, Empathy } \\
\text { ability and Self esteem on middle school } \\
\text { students" aggression according to the sex" }\end{array}$ & $\begin{array}{c}\text { Empathy Ability, Adolescents, } \\
\text { Middle School Students, Overt } \\
\text { Aggression, Differences Between } \\
\text { the Sexes, Aggression, Relational } \\
\text { Aggression, Narcissism, } \\
\text { Perspective-Taking, Self Esteem }\end{array}$ \\
\hline 25 & $\begin{array}{l}\text { Ju-Hwan } \\
\text { Cha, Dong- } \\
\text { Hyuck Lee }\end{array}$ & & $\begin{array}{l}\text { "The Effects of neuroticism and } \\
\text { attachment security on social skills: } \\
\text { Mediating roles of attention control and } \\
\text { empathy" }\end{array}$ & $\begin{array}{c}\text { Empathy, Social Skills, } \\
\text { Adolescents, Neuroticism, } \\
\text { Attachment Security, Attention } \\
\text { Control }\end{array}$ \\
\hline 26 & $\begin{array}{l}\text { Jeong-Ae Ko, } \\
\text { Kyung Park }\end{array}$ & & $\begin{array}{c}\text { "Program Development for Victim } \\
\text { Empathy Enhancing in Juvenile Sexual } \\
\text { Offenders" }\end{array}$ & $\begin{array}{l}\text { Victim Empathy Therapy, } \\
\text { Cognitive Behavioral, } \\
\text { Interpersonal Rreactivity Index, } \\
\text { Juvenile Sexual Offender }\end{array}$ \\
\hline 27 & $\begin{array}{l}\text { Mi -Sun Oh, } \\
\text { Il-Nam Kwon }\end{array}$ & \multirow{3}{*}{2012} & $\begin{array}{l}\text { "The effects of mentor`s empathic ability } \\
\text { on mentoring relationship and mentee`s } \\
\text { self-esteem" }\end{array}$ & $\begin{array}{l}\text { Empathy, Youth Mentoring } \\
\text { Activity, Self-Esteem }\end{array}$ \\
\hline 28 & Na-Min Shin & & $\begin{array}{l}\text { "Empathy and bullying: How are they } \\
\text { related in explaining the types of bullying } \\
\text { participations among adolescents" }\end{array}$ & $\begin{array}{l}\text { Cognitive Empathy, School } \\
\text { Violence, Bullying, Empathy, } \\
\text { Adolescent, Empathic } \\
\text { Understanding, Theory of Mind }\end{array}$ \\
\hline 29 & $\begin{array}{l}\text { In-Young } \\
\text { Kim }\end{array}$ & & $\begin{array}{c}\text { "A Study on the Empathic Leadership of } \\
\text { Youth Workers" }\end{array}$ & $\begin{array}{l}\text { Empathic leadership, Youth } \\
\text { Activity, Youth Worker }\end{array}$ \\
\hline 30 & Yong-Joo Jo & \multirow{3}{*}{2010} & $\begin{array}{c}\text { "Development and Validation of Parental } \\
\text { Empathy Inventory Perceived by } \\
\text { Adolescents" }\end{array}$ & $\begin{array}{c}\text { Cold Emotional Reaction, } \\
\text { Awareness of Emotion, Excessive } \\
\text { Emotional Reaction, Empathic } \\
\text { Emotional Reaction, Parental } \\
\text { Empathy Perceived by } \\
\text { Adolescents, Perspective Taking }\end{array}$ \\
\hline 31 & Sung-Il Kim & & $\begin{array}{l}\text { "Validation of The Emotional Empathy } \\
\text { Scale for Adolescents" }\end{array}$ & $\begin{array}{c}\text { Empathy, Adolescent, } \\
\text { Psychological Test, Test } \\
\text { Validation }\end{array}$ \\
\hline 32 & $\begin{array}{l}\text { Myung-Sun } \\
\text { Chung }\end{array}$ & & $\begin{array}{c}\text { "The Effects of Empathy Training Group } \\
\text { Art Therapy on Adolescents with Histories } \\
\text { of School Violence : Focusing on the } \\
\text { Variables of Emotional Empathy and } \\
\text { Aggression" }\end{array}$ & $\begin{array}{c}\text { Empathy, School Violence, } \\
\text { Adolescent, Aggression, Group } \\
\text { Art Therapy }\end{array}$ \\
\hline
\end{tabular}

이에 본 연구에서는 연구 대상자의 집단 연령 구분에 따라 초기 청소년, 중학생, 고등학생, 중-고등학생, 후기 청소년, 청소년 관련 성인(상담자 및 부모)으로 분류하였고, 집단 설계에 따른 교차 분석을 시행하였으며, 그 결과는 다음 [표 4]와 같다. 
[표 4] 연구 집단 설계에 따른 연령 분류

[Table 4] Age Classification According to the Research Group Designed

\begin{tabular}{|c|c|c|c|c|c|c|c|c|c|}
\hline \multirow{2}{*}{\multicolumn{3}{|c|}{ Division }} & \multicolumn{6}{|c|}{ Age classification } & \multirow{3}{*}{$\begin{array}{r}\text { Total } \\
27 \\
\end{array}$} \\
\hline & & & \multirow{2}{*}{$\begin{array}{c}\text { Early } \\
\text { Youth } \\
2\end{array}$} & \multirow{2}{*}{$\begin{array}{c}\text { Middle } \\
\text { Schooler } \\
6\end{array}$} & \multirow{2}{*}{$\begin{array}{c}\begin{array}{c}\text { High } \\
\text { school } \\
\text { student }\end{array} \\
1\end{array}$} & \multirow{2}{*}{$\begin{array}{c}\text { Middle } \\
\text { School } \\
\text { and High } \\
\text { School }\end{array}$} & \multirow{2}{*}{$\begin{array}{c}\text { Late } \\
\text { Youth }\end{array}$} & \multirow{2}{*}{$\begin{array}{c}\begin{array}{c}\text { Youth } \\
\text { Related } \\
\text { Adults }\end{array} \\
3\end{array}$} & \\
\hline \multirow{8}{*}{$\begin{array}{l}\text { Group } \\
\text { Design }\end{array}$} & \multirow{2}{*}{$\begin{array}{l}\text { Experimental } \\
\text { Group }\end{array}$} & $\mathrm{N}$ & & & & & & & \\
\hline & & $(\%)$ & 6.3 & 18.7 & 3.1 & 37.5 & 9,4 & 9.4 & 84.4 \\
\hline & \multirow{2}{*}{$\begin{array}{l}\text { Experimental- } \\
\text { Control Group }\end{array}$} & $\mathrm{N}$ & 0 & 0 & 0 & 2 & 0 & 0 & 2 \\
\hline & & $(\%)$ & 0.0 & 0.0 & 0.0 & 6.3 & 0.0 & 0.0 & 6.3 \\
\hline & \multirow{2}{*}{$\begin{array}{c}\text { Experimental- } \\
\text { Comparison Group }\end{array}$} & $\mathrm{N}$ & 0 & 0 & 1 & 1 & 0 & 0 & 2 \\
\hline & & $(\%)$ & 0.0 & 0.0 & 3.1 & 3.1 & 0.0 & 0.0 & 6.2 \\
\hline & \multirow{2}{*}{$\begin{array}{l}\text { Experimental- } \\
\text { Comparison- } \\
\text { Control Group }\end{array}$} & $\mathrm{N}$ & 0 & 1 & 0 & 0 & 0 & 0 & 1 \\
\hline & & $(\%)$ & 0.0 & 3.1 & 0.0 & 0.0 & 0.0 & 0.0 & 3.1 \\
\hline & \multirow{2}{*}{ Total } & $\mathrm{N}$ & 2 & 7 & 2 & 15 & 3 & 3 & 32 \\
\hline & & $(\%)$ & 6.3 & 21.8 & 6.2 & 46.9 & 9,4 & 9,4 & 100.0 \\
\hline
\end{tabular}

청소년 상담에서 공감을 연구한 총 32 편(100.0\%) 중에서 집단의 설계는 단일 실험 집단 $(84.4 \%, \mathrm{~N}=27)$ 과 실험-통제 집단 $(6.3 \%, \mathrm{~N}=2)$, 실험-비교 집단 $(6.2 \%, \mathrm{~N}=2)$, 그리고 실험-비교-통제 집단 $(3.1 \%, \mathrm{~N}=1)$ 으로 구성하였다. 단일 실험 집단 설계에서는 초기 청소년 집단 각 2 편 $(6.3 \%)$, 중학생 집단 각 6 편(18.7\%), 고등학생 집단 1 편(3.1\%), 중-고등학생 집단 각 12 편 $(37.5 \%)$, 후기 청소년 집단 각 3 편 $(9.4 \%)$, 청소년 관련 성인(상담자 및 부모) 집단 각 3 편 $(9.4 \%)$ 으로 보고되었다. 또한 실험-통제 집단 설계에서도 중-고등학생 각 2 편 $(6.3 \%)$ 으로 나타났으며, 실험-비교 집단 설계에서는 고등학생 집단 1 편 $(3,1 \%)$, 중고등학생 집단 1 편 $(3,1 \%)$, 그리고 실험-비교-통제 집단 설계에서 중학생 집단 1 편(3.1\%)으로 보고되었다. 청소년 공감 연구 방법의 분류는 다음 [표 5]와 같다.

[표 5] 청소년 공감에 대한 연구방법 분류

[Table 5] Youth Sympathy Research Methods Classification

\begin{tabular}{c|c|c|c|c}
\hline Division & Quantitative Research & Qualitative Research & Literature Research & Total \\
\hline $\mathbf{N}$ & 29 & 2 & 1 & 32 \\
\hline$(\%)$ & 90.6 & 6.3 & 3.1 & 100.0 \\
\hline
\end{tabular}


이처럼 청소년 상담에서 공감을 연구한 총 32 편(100.0\%) 중 29 편 $(90.6 \%)$ 의 논문이 양적 연구 방법을 활용하였고, 2 편 $(6.3 \%)$ 의 논문은 질적 연구 방법으로 나타났으며, 1 편 $(3.1 \%)$ 의 논문은 문헌 연구 방법으로 보고되었다. 연구 방법에 따른 청소년 공감 연구 분류는 다음 [표 6]와 같다.

[표 6] 연구 방법에 따른 청소년 공감 연구 분류

[Table 6] Youth Empathy Studies Classified according to the Research Method

\begin{tabular}{c|c|c|c|c|c|c|c|c}
\hline $\begin{array}{c}\text { Divisio } \\
\mathbf{n}\end{array}$ & $\begin{array}{c}\text { Mediation } \\
\text { Effect } \\
\text { Research }\end{array}$ & $\begin{array}{c}\text { Literature } \\
\text { Research }\end{array}$ & $\begin{array}{c}\text { Phenomenology } \\
\text { Research }\end{array}$ & $\begin{array}{c}\text { Case } \\
\text { Research }\end{array}$ & $\begin{array}{c}\text { Program } \\
\text { Development } \\
\text { Research }\end{array}$ & $\begin{array}{c}\text { Program } \\
\text { Effectiveness } \\
\text { Research }\end{array}$ & $\begin{array}{c}\text { Scale paper } \\
\text { Development } \\
\text { Research }\end{array}$ & Total \\
\hline $\mathbf{N}$ & 20 & 1 & 1 & 1 & 2 & 4 & 3 & 32 \\
\hline$(\%)$ & 62.5 & 3.1 & 3.1 & 3.1 & 6.3 & 12.5 & 9.4 & 100.0 \\
\hline
\end{tabular}

특히 전체 ‘청소년 공감' , ‘청소년 공감 예술' 을 주제어 키워드로 한 총 32 편(100.0\%)의 연구 방법의 결과, 매개효과 연구 20 편(78.1\%), 문헌연구 1 편 $(3.1 \%)$, 현상학적 연구 1 편(3.1\%), 사례연구 1 편(3.1\%), 프로그램 개발 연구 2 편(6.3\%), 프로그램 효과 연구 4 편 $(\%)$, 척도지 개발 연구 3 편 $(9.4 \%)$ 으로 확인되었으며, 예술매체를 활용한 공감 연구 분류는 다음 [표 7]과 같다.

[표 7] 예술매체를 활용한 공감 연구 분류

[Table 7] Empathy Research Frequently Utilizing the Medium of Arts

\begin{tabular}{c|c|c|c}
\hline Division & Empathy Research & Arts Therapy Empathy Research & Total \\
\hline $\mathbf{N}$ & 28 & 4 & 32 \\
\hline$(\boldsymbol{\%})$ & 87.5 & 12.5 & 100.0 \\
\hline
\end{tabular}

이때 예술매체를 활용한 공감 연구는 전체 논문 32편(100.0\%) 중에서 총 4편(12.5\%)으로 나타났으며, 통합예술치료(1편), 집단미술치료(2편), 인지행동치료(1편) 프로그램을 확인할 수 있었다. 전체 논문 주제어에 따른 공감 키워드 분류는 다음 [표 8]과 같다.

[표 8] 전체 논문 주제어에 따른 공감 키워드 분류

[Table 8] Totall Research Key Word Frequency with Empathy

\begin{tabular}{c|c|c|c}
\hline Division & Empathy Research Key Word & Other Research Key Words & Totall Research Key Words \\
\hline $\mathbf{N}$ & 39 & 111 & 150 \\
\hline$(\%)$ & 26.0 & 74.0 & 100.0 \\
\hline
\end{tabular}

청소년 상담에서 공감 연구의 전체 논문 32 편(100.0\%) 중 전체 주제어 키워드는 총 150 개 $(100.0 \%)$ 로 확인되었다. 이 중 공감이 들어간 주제어 키워드 논문은 총 39 개(26.0\%)이고, 그 외 주제어 키워드 논문은 111개(74.0\%)로 나타났으며, 공감 단어가 들어간 주제어 분류는 다음 [표 9]과 같다. 
[표 9] 공감 단어가 들어간 주제어 분류

[Table 9] Key Word Frequency with Empathy

\begin{tabular}{|c|c|c|c|}
\hline NO & Empathy Research Key Word & $\mathbf{N}$ & $(\%)$ \\
\hline 1 & Empathy & 13 & 33.3 \\
\hline 2 & Sympathy & 9 & 23.1 \\
\hline 3 & Empathy Scale & 2 & 5.1 \\
\hline 4 & Emotional Empathy & 3 & 7.6 \\
\hline 5 & Cognitive Empathy & 2 & 5.1 \\
\hline 6 & Working Environment & 1 & 2.6 \\
\hline 7 & Cultural Empathy & 1 & 2.6 \\
\hline 8 & Parental Empathy & 3 & 7.6 \\
\hline 9 & Cognitive Behavioral & 1 & 2.6 \\
\hline 10 & Victim Empathy therapy & 1 & 2.6 \\
\hline 11 & Empathic Leadership & 1 & 2.6 \\
\hline 12 & Empathic Understanding & 1 & 2.6 \\
\hline 13 & Empathy Training Program & 1 & 2.6 \\
\hline \multicolumn{2}{|r|}{ Total Key Word } & 39 & 100.0 \\
\hline
\end{tabular}

청소년 상담에서 공감 연구의 전체 논문 32 편 $(100.0 \%)$ 중 공감 단어가 들어간 논문 주제어 키워드는 총 39개(100.0\%)로 확인되었다. 그 결과, 공감 능력 13개(33.3\%), 공감 9 개 $(23.1 \%)$, 공감 척도 2 개 $(5.1 \%)$, 정서적 공감 3 개 $(7.6 \%)$, 인지적 공감 2 개 $(5.1 \%)$, 공감 피로 1 개(02.6\%), 문화적 공감 1 개(2.6\%), 부모 공감 3 개(7.6\%), 대인간 공감 1 개(2.6\%), 피해자 공감 1 개(2.6\%), 공감적 리더십 1 개(2.6\%), 공감적 이해 1 개(2.6\%), 공감 능력 향상프로그램 1 개(2.6\%)로 나타남을 확인할 수 있었다. 한편, 공감을 중심으로 선행된 예술치료 연구들을 상세히 살펴보면, 청소년 상담 과정에서 상담자의 공감을 지각하는 내담자 경험에 대한 현상학적 질적 연구[22] 소년원 재소 비행청소년을 대상으로 인지 행동이론을 활용하여 집단미술치료 프로그램이 부정적·자동적 사고와 공감 능력에 대한 정적 상관관계 연구[23], 그리고 집단미술치료 공감 훈련 프로그램을 통하여 학교폭력 청소년들의 정서 공감 향상과 공격성 수준의 감소 변화에 대한 효과성을 규명한 연구[24], 통합예술치료 프로그램 안에서 발달장애청소년의 공감 능력 향상을 다루는 질적 연구[25]로 나타남을 알 수 있었다.

특히, 최미경과 이서영[26]은 공감을 중심으로 학교폭력 예방교육 프로그램 안에서 학교 부적응 청소년의 학교폭력 인식과 태도 변화 및 공감 능력에 관한 효과를 보고하였으며, 고문정[2]은 상담자의 공감 나누기 체험에서 내담자, 관찰자, 상담자라는 다양한 관점에서 공감에 대한 내용을 어떻게 탐색하고 인식하며, 그 결과가 무엇인지 범주화하고 개념화하였다. 또한 김지민과 임영식[27]은 청소년 상담 과정에서 내담자가 지각하는 초기 상담에 대한 기대, 부정적 정서와 공감, 작업 동맹 간의 관계를 규명하였다. 이창식과 장하영[28]은 청소년 공감 능력에 대한 일반적 특성 중에서는 성별과 학교 급에 따라 
차이가 나타나고 자아존중감과 공격성 그리고 공감 능력 모두에서 부적 상관관계를 보고하였다. 엄서영, 장수미와 허성희[29]는 여자 청소년을 대상으로 성 비행 피해자의 공감 능력 향상을 활용한 프로그램을 개발하여 그 효과성을 입증하였다. 마지막으로 김유성과 민희정[30]은 후기 청소년기 대학생을 대상으로 자기 자비와 메타 인지의 관계를 검증하였고, 자기효능감과 인지적 공감의 매개효과를 제시하였다. 이처럼 공감은 청소년의 일상생활과 깊은 연관성이 있으며, 사회적 관계에서 경험하게 되는 조화로운 역할과 소통은 대인관계를 촉진시키고 행복감을 경험하게 한다.

\section{5. 결론 및 제언}

본 연구는 청소년 상담에서 예술매체를 활용한 공감 연구동향 선행연구를 기초로 예술이 지닌 다양한 치료적 기제 기능의 장점과 의미를 재확인하였다. 청소년 상담에서 치료적 변화를 위한 다양한 목적에 따라 예술매체를 활용한 공감 이론의 가능성을 탐색하였다는데 의의가 있다. 공감 이론을 중심으로 이루어진 청소년 상담연구에서 탈피하여 다양한 예술매체 접근법을 고찰했다는 점에서 의의가 있다. 그리고 청소년 상담에서 예술매체를 활용한 공감과 관련된 선행연구들을 RISS에서 $\mathrm{KCI}$ 등재된 학술지 논문을 중심으로

'청소년 공감' , ‘청소년 공감 예술' 이라는 주제어 키워드로 총 78 편의 논문을 검색하였다는데 의의가 있다. 9 개의 학술지 영역에서 주제어 키워드로 '공감' 이 포함된 논문은 총 32 편(100.00\%)으로 확인되었는데 그 결과, 2010년 3편(9.4\%), 2012년 3편(9.4\%), 2013년 3편(9.4\%), 2014년 1편(3.1\%), 2015년 6편(18.7\%), 2017년 3편(9.4\%), 2018년 7편(21.9\%), 2019년 4편(12.5\%), 2020년 1편(3.1\%), 2021년 1편(3.1\%)으로 연도별 청소년 공감 논문 현황을 파악하였다는 것에 큰 의미가 있다. 이는 상담학 분야 주요 학술지를 중심으로 공감 연구 동향을 검토한 하소영[4]의 연구와 명상 프로그램의 효과성 동향을 살펴본 김희진과 이미선[21]의 연구가 부분적으로 일치한다.

특히 청소년 상담에서 공감을 연구한 총 32 편(100.0\%) 중 집단의 연령 구분을 초기 청소년, 중학생, 고등학생, 중-고등학생, 후기 청소년, 청소년 관련 성인(상담자 및 부모)으로 분류하였으며, 집단 설계는 단일 실험 집단 $(84.4 \%, \mathrm{~N}=27)$ 과 실험-통제 집단 $(6.3 \%, \mathrm{~N}=2)$, 실험-비교 집단 $(6.2 \%, \mathrm{~N}=2)$, 그리고 실험-비교-통제 집단 $(3.1 \%$, $\mathrm{N}=1)$ 으로 보고되었으며, 양적 연구가 29편(90.6\%)이며, 질적 연구가 2편(6.3\%), 그리고 문헌 연구가 1 편(3.1\%)의 결과를 확인할 수 있었다. 매개효과 연구 20 편(78.1\%), 문헌 연구 1 편(3.1\%), 현상학적 연구 1 편(3.1\%), 사례연구 1 편(3.1\%), 프로그램 개발 연구 2 편(6.3\%), 프로그램 효과 연구 4 편(12.5\%), 척도지 개발 연구 3 편(9.4\%)의 결과와 예술매체를 활용한 예술치료 관련 연구는 전체 논문 32편(100.0\%) 중 총 4편(12.5\%)으로 매우 미비하였음을 확인할 수 있었다. 이는 청소년 상담에서 예술매체를 활용한 공감 연구가 한정적이고 부족한 실정을 파악하였다는 것에 큰 의의가 있다. 그러므로 다양한 예술매체를 활용하여 청소년 대상자의 특성과 연령 및 발달단계를 고려한 대상별 공감 프로그램 구성이 요구된다.

또한 청소년 공감 연구의 전체 논문 32 편(100.0\%) 중에 전체 주제어 키워드는 총 150 개(100.0\%)로 공감이 들어간 주제어 키워드는 39개(26.0\%)이며, 그 외 나머지 주제어 키워드는 111 개(74.0\%)의 결과가 나타남을 알 수 있었다. 더불어 공감이 들어간 주제어 키워드 논문 39개(100.0\%)에 따른 분류 결과, 공감 능력 13개(33.3\%), 공감 9개(23.1\%), 공감 척도 2 개 $(5.1 \%)$, 정서적 공감 3 개 $(7.6 \%)$, 인지적 공감 2 개 $(5.1 \%)$, 공감 피로 
1 개(02.6\%), 문화적 공감 1 개(2.6\%), 부모 공감 3 개(7.6\%), 대인간 공감 1 개(2.6\%), 피해자 공감 1 개(2.6\%), 공감적 리더십 1 개(2.6\%), 공감적 이해 1 개(2.6\%), 공감 능력 향상프로그램 1 개(2.6\%)는 13 개 분류에 따라 공감 주제어 키워드 빈도 결과를 도출하였다. 이러한 결과는 하소영[4]의 연구와 김희진과 이미선[21]의 연구에서 나타난 결과와 부분적으로 맥을 같이하며, 공감 연구에서 연구의 주제어 키워드가 다양하지 못하고 매우 제한적임을 시사할 수 있다. 반면, 예술매체와 관련된 공감 주제어 키워드가 없음을 확인하였다는 것에 의의가 있다. 이는 청소년 상담에서 예술매체를 활용한 치료적 개입으로서의 다양한 '공감' 주제어 키워드 연구가 필요함을 시사한다. 앞서 제시한 바와 같이 예술매체를 활용한 공감에 관한 연구 수는 매우 한정적이고, 충분하지 않다는 연구결과를 기초로 하여 추후 연구에서는 치료적 도구로서 예술이 지닌 효과성을 검증하기 위한 공감 연구의 보급이 시급하다고 본다.

따라서 본 연구를 참고하여 다양한 예술매체를 적극적으로 활용한 청소년 공감 연구를 진행할 것을 제언하는 바이며, 다양한 영역의 대상과 주제에 관한 키워드 등의 경험적 임상 연구들이 추가적으로 시행되어야 할 것이다. 향후 청소년 공감 프로그램의 과학적이고 체계적인 검증과 효과적인 분석을 위한 자료로 활용되기를 기대한다.

\section{References}

[1] S. Baron-Cohen, J. Richler, D. Bisarya, N. Gurunathan, S. Wheelwright, The systemizing quotient: an investigation of adults with Asperger syndrome or high-functioning autism, and normal sex differences, Philosophical Transactions of the Royal Society of London Series B: Biological Sciences, (2003), Vol.358, No.1430, pp.361-374, DOI: $10.1098 /$ rstb.2002.1206

[2] M. J. Ko, A Qualitative Study on Counselors' Experiences of Sharing Empathy, Korea Journal of Counseling, (2016), Vol.17, No.2, pp.1-23, DOI : 10.15703/kjc.17.2.201604.1

[3] J. S. Park, (The) relationships among fifth graders' empathy, peer relationship, and self-regulation, Kyunghee University, Master's thesis, (2010)

[4] S. Y. Ha, A Study on Empathy in Counseling: Focused on the major academic journals, Journal of Counseling Education $\begin{array}{llll}\text { Research, } & \text { (2019), } & \text { Vol.2, } & \text { No.2. }\end{array}$ https://scholar.dkyobobook.co.kr/searchDetail.laf?barcode $=4050027615389$

[5] S. A. Kim, J. Ha, The Mediating Effect of Soothing Ability and Perceived Social Support In The Relation between Object Relation Level and Empathic Ability of Adolescence, Korean Journal Of Counseling And Psychotherapy, (2014), Vol.26, No.2, pp.503-524, UCI : G704-000523.2014.26.2.011

[6] Y. Y. Hong, Y. S. Kim, Development and Validation of the Adolescent Empathy Scale, Korean Journal of Youth Studies, (2015), Vol.22, No.8, pp.159-183, UCI : G704-000387.2015.22.8.007

[7] J. E. Park, E. H. Lee, Adolescents` Insecure Attachments and Problem Behaviors: The Moderating Role of Empathic Ability, Korean Journal Of Counseling And Psychotherapy, (2008), Vol.20, No.2, pp.369-389, UCI : G704000523.2008.20.2.009

[8] G. M. Hong, Relationship between Empathy level and Social competence of adolescents, Sookmyung Womens's University, Master's thesis, (2004)

[9] C. R. Rogers, The necessary and sufficient conditions of therapeutic personality change, Journal of Consulting Psychology, (1957), Vol.21, No.2, pp.95-103, DOI : https://doi.org/10.1037/h0045357

[10] J. F. Dovidio, Helping behavior and altruism: An empirical and conceptual overview, Advances in Experimental Social Psychology, (1984), Vol.17, pp.361-427, DOI : 10.1016/S0065-2601(08)60123-9 
[11] R. C. Mackay, What is empathy?, Empathy in the helping relationship, Springer, (1990)

[12] M. S. Chung, A Validation Study of the Korean-Balanced Emotional Empathy Scale, Korea Journal of Counseling, (2012), Vol.13, No.4, pp.1781-1797, DOI : 10.15703/kjc.13.4.201208.1781

[13] Y. H. Kim, J. S. Kim, Development and Validation of Empathy Scale, Korea Journal of Counseling, (2017), Vol.18, No.5, pp.61-84, DOI : 10.15703/kjc.18.5.201710.61

[14] S. H. Hur, Development of Scale and Program for Empathy Capability, Daejeon University, Doctoral Dissertation, (2017)

[15] S. H. King, M. J. Holosko, The development and initial validation of the empathy scale for social workers, Research on Social Work Practice, (2012), Vol.22, No.2, pp.174-185, DOI : 10.1177/1049731511417136

[16] S. H. King., The structure of empathy in social work practice, Journal of Human Behavior in the Social Environment, (2011), Vol.21, No.6, pp.679-695, DOI : 10.1080/10911359.2011.583516

[17] E. A. Segal, Social Empathy: A Model Built on Empathy, Contextual Understanding, and Social Responsibility That Promotes Social Justice, Journal of Social Service Research, (2011), Vol.37, No.3, pp.266-277, DOI : $10.1080 / 01488376.2011 .564040$

[18] W. F. Sun, H. J. So, Arts Therapy, Park Young Story, (2019)

[19] Y. J. Hong, Intergrative arts therapy, Hakjisa, (2017)

[20] S. Y. Park, A Study on the Countertransference of Therapists in Integrated Arts Therapy for Emotional Self-reliance of At-risk Youth, Dongduk Women's University, Doctoral Dissertation, (2021)

[21] H. J. Kim, M. S. Lee, The Analysis of Research Trends on Effectiveness of the Medication Program: Focusing on Published in 2000-2020, Culture and Convergence (Culture and Convergence), (2021), Vol.43, No.2, pp.753-774.

[22] Y. H. Oh, A Phenomenological Research on Perception of Adolescent clients about Counselor's Empathy, The Korea Journal of Youth Counseling, (2015), Vol.23, No.2, pp.299-319, DOI : 10.35151/kyci.2015.23.2.014

[23] S. J. Oh, S. M. Choi, The Effects of Group Art Therapy Based on Cognitive Behavior Theory on Automatic Thought, and Empathy of Juvenile Delinquents, Journal of Welfare for the correction, (2015), No.38, pp.121-146, UCI : G704SER000002276.2015..38.001

[24] M. S. Chung, The Effects of Empathy Training Group Art Therapy on Adolescents with Histories of School Violence : Focusing on the Variables of Emotional Empathy and Aggression, Studies on Korean Youth, (2010), Vol.21, No. 4, pp.51-77, UCI : G704-001385.2010.21.4.004

[25] M. J. Shin, Y. K. Joo, A Qualitative Case Study on Integrated Art Therapy Program to Improve Empathy Ability of Adolescents with Developmental Disabilities, Journal of Educational Innovation Research, (2018), Vol.28, No.4, pp.413-440, DOI : 10.21024/pnuedi.28.4.201812.413

[26] M. K. Choi, S. Y. Lee, A Study on Empathy Ability, Awareness \& Attitude Change through Empathy Centered-School Violence Prevention Education for Non-Adapted Student, Korean Journal of youth welfare, (2015), Vol.17, No.1, pp.75-96, UCI : G704-002137.2015.17.1.016

[27] J. M. Kim, Y. S. Lim, The influence on client's expectations about counseling(EAC), perception of adolescent about counselor's empathy, negative emotion on working alliance in early sessions in counseling, Korean Journal of Youth Studies, (2018), Vol.25, No.12, pp.281-304, DOI : 10.21509/KJYS.2018.12.25.12.281

[28] C. S. Lee, H. Y. Jang, Moderating Effect of Empathy on the Relationship between Self-esteem and Aggression of Adolescents, Journal of Digital Convergence, (2018), Vol.16, No.1, pp.47-53, DOI : 10.14400/JDC.2018.16.1.047

[29] S. Y. Eom, S. M. Jang, S. H. Heo, The Development and Effects of the Empathy Enhancement Program for Females Adolescent with Sexual Misconducts, Korean Journal of Youth Studies, (2019), Vol.26, No.8, pp.79-107, DOI : 10.21509/KJYS.2019.08.26.8.79

[30] Y. S. Kim, H. J. Min, The Mediating Effects of Self-Efficacy and Cognitive Empathy in the Relationships between College Students' Self-Compassion and Meta-Cognition, Korean Journal of Youth Studies, (2021), Vol.28, No.3. pp.339-365, DOI : 10.21509/KJYS.2021.03.28.3.339 